\title{
Plasma Synthesis of Facetted Nickel nano-Ferrites with Controlled Stoichiometry
}

\author{
S. Bastien ${ }^{1}$, C. Ricolleau ${ }^{2}$, N. Braidy ${ }^{1}$.
}

${ }^{1}$ Chemical and Biotechnological Engineering, Université de Sherbrooke. 2500 Boul. de l'Université, Sherbrooke, Québec, Canada J1H 2R1.

${ }^{2}$ Laboratoire Matériaux et Phénomènes Quantiques, CNRS-UMR 7162, Université Paris Diderot Paris 7, Case Courrier 7021, 75205 Paris Cedex 13, France.

Nickel ferrite is a typical inverse spinel with the general chemical formula $\mathrm{Ni}_{\mathrm{x}} \mathrm{Fe}_{3-\mathrm{x}} \mathrm{O}_{4}$. Its chemical composition is highly malleable, allowing for the modulation of the magnetic and chemical properties of the structure. Such flexibility related to these properties can then be exploited in applications such as hyperthermia or catalytic reforming of hydrocarbons.

According to the pseudo-equilibrium $\mathrm{Fe}_{2} \mathrm{O}_{3}-\mathrm{NiO}$ phase diagram [1], $\mathrm{Ni}_{\mathrm{x}} \mathrm{Fe}_{3-\mathrm{x}} \mathrm{O}_{4}$ is only stable at room temperature when $x=1$. The spinel phase is stable for $0 \leq x \leq 1$ at high temperatures $\left(>800^{\circ} \mathrm{C}\right)$, whereas values of $x>1$ are thermodynamically impossible; the excess $\mathrm{Ni}$ will instead separate into a secondary $(\mathrm{Ni}, \mathrm{Fe}) \mathrm{O}$ phase. Co-precipitation, sol-gel and ball milling are common methods to prepare Ni-ferrites although the control of stoichiometry is challenging. With either of these methods, an annealing step is required to achieve the optimal magnetic or catalytic properties which inevitably promotes the segregation of $\mathrm{Fe}_{2} \mathrm{O}_{3}$ and sintering.

Here, we demonstrate the possibility of producing Ni-ferrites nanocrystals (NCs) with controlled stoichiometry using solution spray induction plasma [2]. Ni and Fe nitrates (with Ni:Fe ratio 0, 1:11, $1: 5,1: 2$ and 1:1) were sprayed in the vicinity of a radio-frequency induced plasma (PL50 torch Tekna Plasma Systems inc., $30 \mathrm{~kW}, 200$ Torr $\mathrm{O}_{2}+\mathrm{Ar}$ ). X-ray fluorescence measurements reveal that the $\mathrm{Ni}$-ferrites produced exhibit a global $\mathrm{Ni}$ to $\mathrm{Fe}$ ratio equal to the nominal ratio of feed precursors. Rietveld refinement on X-ray diffraction patterns confirm the exclusive crystal structure of -ferrite for the first four composition, and a mixture of $\mathrm{NiFe}_{2} \mathrm{O}_{4}$ and $\left(\mathrm{Ni}_{0.9}, \mathrm{Fe}_{0.1}\right) \mathrm{O}$ in the 1:1 $\mathrm{Ni}$ :Fe sample. Transmission electron microscopy (TEM) studies on these nanocrystals were conducted using an ARM JEOL TEM operating at $200 \mathrm{kV}$ equipped with a Gatan ${ }^{\mathrm{TM}}$ GIF Quantum ER spectrometer. The samples were prepared by leaving a drop of a suspension of Ni-ferrite NCs to dry on a holey C-coated Cu TEM grid under a magnetic field to ensure optimal dispersion.

Fig. 1a and 1c exhibit Sobel-filtered images of high-angle annular dark field micrographs of two types of NCs (1:2 and 1:1 Ni:Fe ratio, respectively). These NCs adopt the shape of truncated octahedrons, with sizes ranging from 6 to $160 \mathrm{~nm}$. High resolution electron microscopy studies confirm that the $\{111\}$ and $\{100\}$ facets are exposed, as expected from the equilibrium Wulff shape of FCC crystals. The shape and particle size distribution of NCs are the same for all samples, regardless of stoichiometry.

Intra-particular variations of the chemical composition of the NCs were evaluated by performing linescans with an electron energy-loss spectrometer and measuring the O K, Fe L23 and Ni L23 edges. For the 1:2 Ni:Fe sample, the linescan exhibits uniform composition (Fig. 1b) within an individual NC, whereas samples with excess $\mathrm{Ni}(1: 1 \mathrm{Ni}: \mathrm{Fe})$ show a segregation of a Ni-rich phase on the edge of the NC (Fig. 1d). Cs-corrected HREM micrograph of a NC from this sample (Fig. 2) reveals the two distinct crystallographic phases that were detected with Rietveld refinement. The core corresponds to $\mathrm{NiFe}_{2} \mathrm{O}_{4}$ in the $<103>$ orientation while the exterior was assigned to the $\left(\mathrm{Ni}_{0.9}, \mathrm{Fe}_{0.1}\right) \mathrm{O}$ phase. The absence of the (200) planes of $\mathrm{NiFe}_{2} \mathrm{O}_{4}$ on the edge of the particle in Fig. 2a suggests that $\left(\mathrm{Ni}_{0.9}, \mathrm{Fe}_{0.1}\right) \mathrm{O}$ covers both types of facets of the NCs. The monoxide layer appears thicker 
on $\{100\}$ facets of the Ni-ferrite than the layer on the $\{111\}$ facets, which suggests a difference in the nature of the respective interfaces.

The TEM observations show that facetted Ni-ferrites NCs can be synthesized by plasma spray deposition with well controlled stoichiometries beyond the solubility range predicted by the equilibrium phase diagram. Thru-focus images and further HREM lattice simulation are required to determine the nature of the two type of interface within individual NCs of the 1:1 Ni:Fe sample.
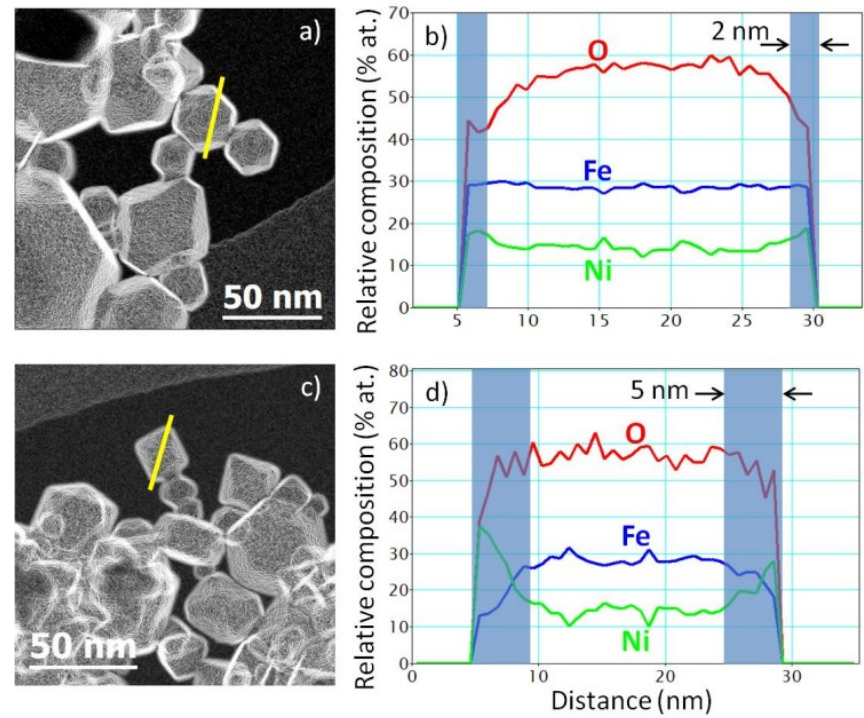

Fig. 1 Sobel-filtered HAADF micrograph (a) and corresponding EELS linescan of chemical composition measured with EELS for the 1:2 Ni:Fe sample (b). Similar data for the 1:1 Ni:Fe sample (c and $d)$.
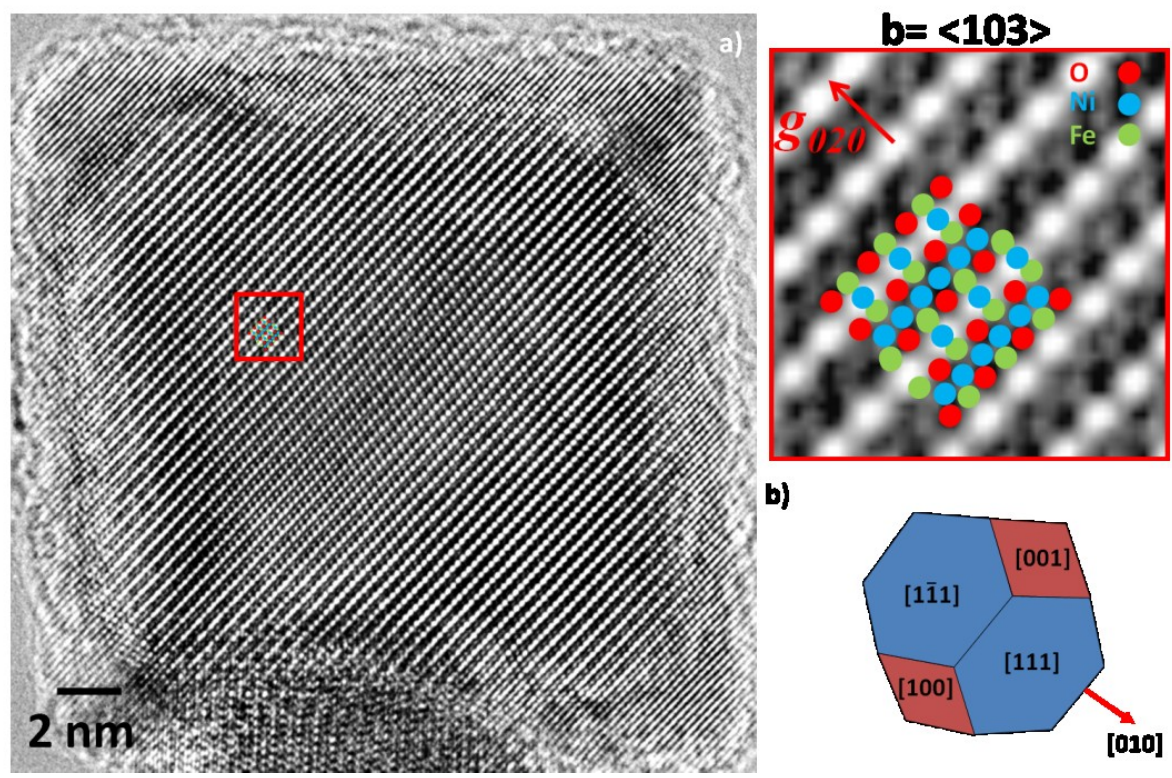

Fig. 2 Objective Cs-corrected TEM micrograph of a single NC for the 1:1 Ni:Fe sample (a). Representation of a truncated octahedron in the $<103>$ direction (b).

\section{References}

[1] M.A. Rhamdhani et al, Metall. Mater. Trans. B. 39 B (2008), p. 690.

[2] S. Bastien and N. Braidy. J. Appl. Phys. 114 (2013), p. 214303 